3. International Committee for the Classification of Retinopathy of Prematurity. An international classification of retinopathy of prematurity. Arch Ophthalmol 1987;105:906-12.

4. Santamarina-Fojo S, Brewer HB. The familial hyperchylomicronaemia syndrome. JAMA 1991;265:904-8.

5. Winder F, Dodson PM, Galton DJ. Ophthalmic complications of the hypertriglyceridaemias. Trans Ophthalmol Soc UK 1980;100:119-22.

Sir,

\section{An Unusual Case of Acute Idiopathic Blind Spot Enlargement Syndrome}

We read with great interest reports suggesting multiple evanescent white dot syndrome (MEWDS), multifocal choroiditis (pseudo-presumed ocular histoplasmosis) and acute macular neuroretinopathy (AMN) are linked by the acute idiopathic blind spot enlargement syndrome (AIBES). ${ }^{1-3} \mathrm{We}$ present a case of AIBES that was preceded by an episode of acute posterior multifocal placoid pigment epitheliopathy (APMPPE) 4 years earlier.

The patient, a healthy 51-year-old woman, presented in April 1991 with a 6-day history of stationary floaters, described as 'like leaves on a tree', affecting her left eye. She denied any preceding 'flu-like illness. On examination, best corrected Snellen acuities were $6 / 6$ in the right eye and 6/36 in the left. Pupil reactions were normal. A very mild anterior uveitis was noted. Slit lamp biomicroscopic examination of the fundus showed multiple post-equatorial circumscribed, flat, greyish-white lesions at the level of the retinal pigment epithelium. These lesions varied from onethird to one-half of a disc diameter in size. Fluorescein angiography showed early blockage of background choroidal fluorescence with late staining of the lesions (Fig. 1). A diagnosis of APMPPE was made and over the ensuing 18 months left eye acuity improved to $6 / 6$ and the fundal lesions disappeared. The patient re-

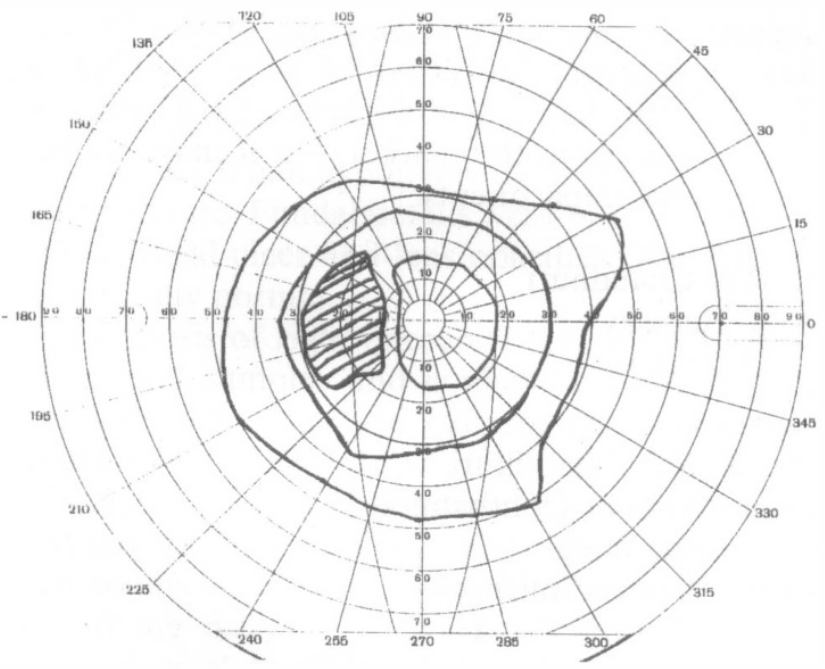

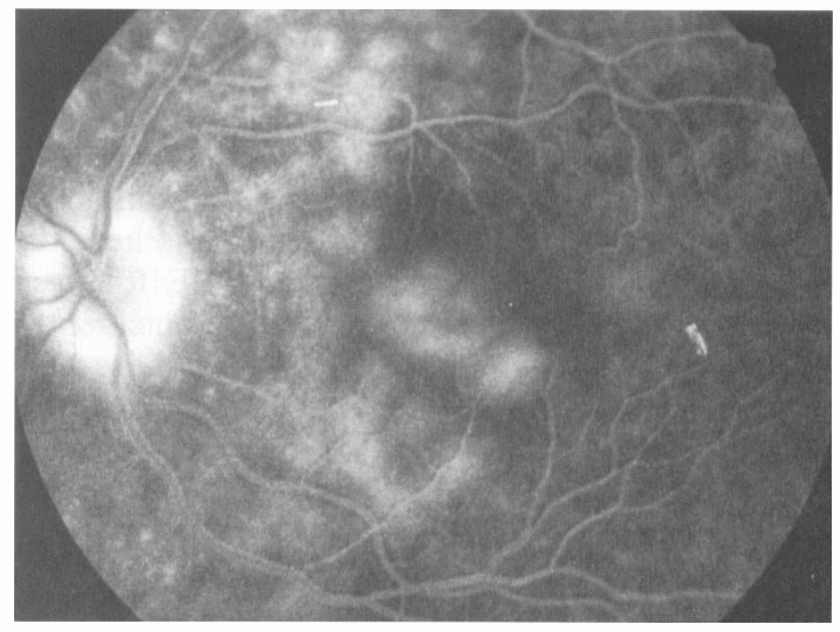

Fig. 1. Late fluorescein frame, demonstrating late staining of lesions, at initial presentation in April 1991.

presented in May 1995 complaining of a 7-week history of a 'whirling' photopsia affecting the left temporal field. We noted the absence of preceding 'flu-like illness. Examination revealed corrected Snellen acuities of $6 / 6$ in the right eye and $6 / 6-1$ in the left. Pupil reactions were normal, as was the anterior segment examination. Fundus examination revealed a normal appearance in both eyes. In particular no white dots or macular granularity were noted. We felt that the history of positive visual phenomena coupled with a normal fundal appearance suggested AIBES. Goldmann visual fields revealed a $20^{\circ}$ enlarged blind spot with steep margins in the left field (Fig. 2), thus confirming our suspicion. At her last review in February 1996 the patient's symptoms had improved; however, her visual fields remain unchanged.

To our knowledge this is the first case of AIBES preceded by APMPPE in the same eye. Many authors including $\mathrm{Gass}^{2}$ feel that MEWDS is a subset of AIBES. Various authors describe evidence

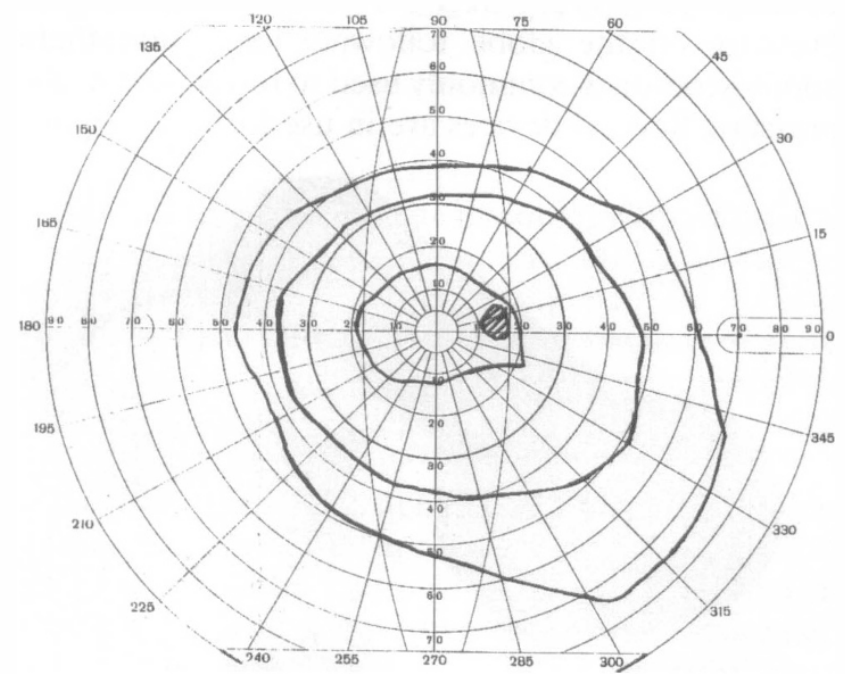

Fig. 2. Goldmann visual fields at second presentation in May 1995. Note the grossly enlarged blind spot in the left visual field. 
suggesting clinicopathological linkage between AIBES, AMN and multifocal choroiditis. ${ }^{1-3}$ It is interesting to note that AIBES/MEWDS is thought to result from pathology at the level of the outer retina whereas APMPPE is thought to result from retinal pigment epithelial disease or choroidal ischaemia. ${ }^{4}$ The occurrence of APMPPE followed by AIBES in our case is thus probably coincidental, but the possibility of a link between these two conditions cannot be excluded.

\section{S. U. Rehman, FRCOphth \\ W. H. Woon, FRCOphth}

Department of Ophthalmology

Leeds General Infirmary

Clarendon Wing

Belmont Grove

Leeds LS2 9NS

UK

Correspondence to:

Mr H. Woon

\section{References}

1. Singh K, et al. Acute idiopathic blind spot enlargement: a spectrum of disease. Ophthalmology 1991;98:497-502.

2. Callanan D, Gass JD. Multifocal choroiditis and choroidal neovascularisation associated with the multiple evanescent white dot and acute idiopathic blind spot enlargement syndrome. Ophthalmology 1992;99: 1678-85.

3. Khorram KD, et al. Blind spot enlargement as a manifestation of multifocal choroiditis. Arch Ophthalmol 1991;109:1403-7.

4. Howe LJ, Woon HW, Graham EM, Fritzke F, Bhandari A, et al. Choroidal hypoperfusion in acute posterior multifocal placoid pigment epitheliopathy: an indocyanine green angiography study. Ophthalmology 1995;102: 790-8.

Sir,

\section{Leakage of Mercury from a McIntyre Bag}

Pressure on the globe following local anaesthetic administration is commonly used to lower intraocular pressure. Several devices are in use for this purpose,

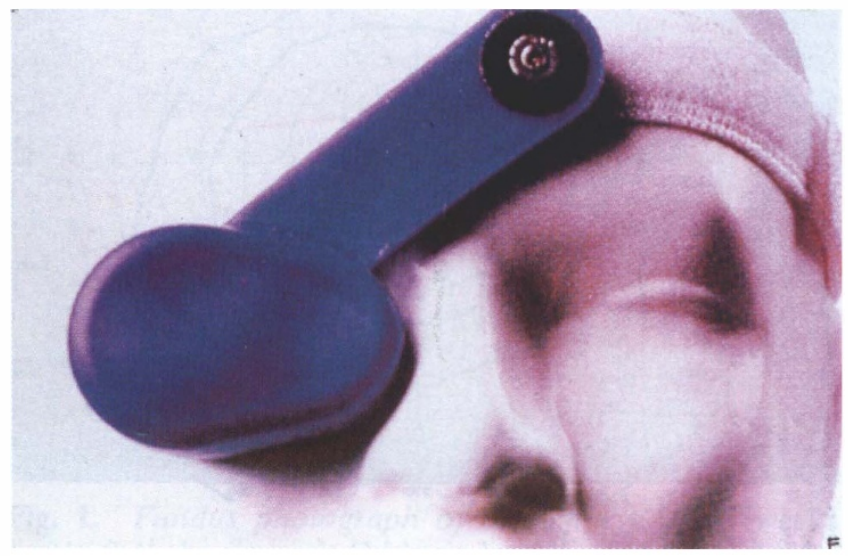

Fig. 1. McIntyre Bag of Mercury.

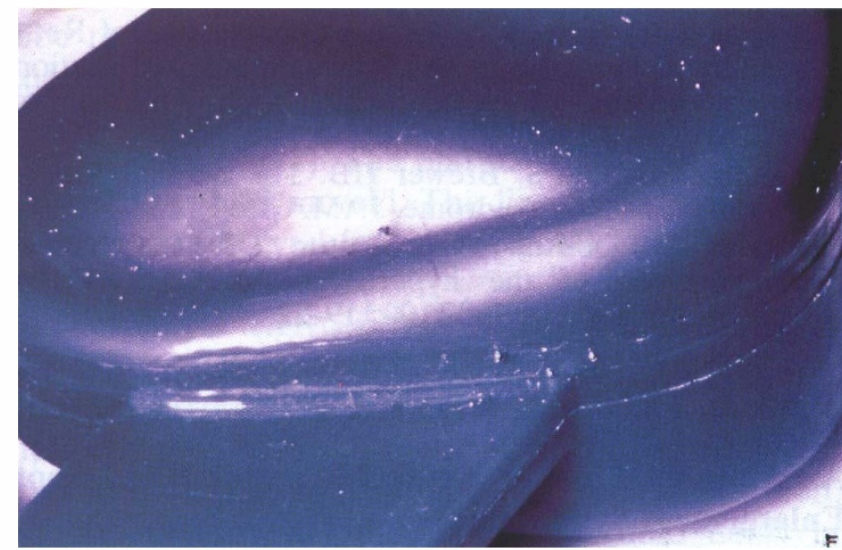

Fig. 2. Leakage of mercury and site of penetration.

including the McIntyre Bag of Mercury (Fig. 1), Honan's Balloon System with a safety valve and the McIntyre Oculo-pressor (made of a high-density solid alloy). We report a case of leakage of mercury from such a device.

\section{Case Report}

A 64-year-old woman was administered a peribulbar local anaesthetic injection for routine cataract surgery. The McIntyre Bag of Mercury was used after the injection. There was leakage of mercury from the device onto the eye and face of the patient. The mercury was removed from the eye and face using pipettes and gauze before liberal irrigation with saline. Subsequent examination of the mercury bag revealed a site of prior needle-stick penetration (Fig. 2).

\section{Comment}

Mercury toxicity is known to cause lenticular and corneal deposits. ${ }^{1}$ However, its more severe systemic effects are well known. ${ }^{2}$ In order to eliminate the risks of exposure to this hazardous metal our department has since ceased use of the McIntyre Bag of Mercury in favour of other oculopressor devices. The bag's manufacturers have withdrawn this product from the market, but it may still be in use in some departments.

N. Ray-Chaudhuri

A. S. Ramsay

N. G. Ziakas

Correspondence to:

Mrs N. Ray-Chaudhuri

Department of Ophthalmology

Claremont Wing

Royal Victoria Infirmary

Queen Victoria Road

Newcastle upon Tyne NE1 4LP

UK

Fax: (+44) 01912275246 